\title{
The immunogenicity and safety of different COVID- 19 booster vaccination following CoronaVac or ChAd0x1 nCoV-19 primary series
}

Nasikarn Angkasekwinai

Faculty of Medicine Siriraj Hospital, Mahidol University https://orcid.org/0000-0001-5723-4877

Suvimol Niyomnaitham

Siriraj Hospital

Jaturon Sewatanon

Siriraj Hospital

Supaporn Phumiamorn

Department of Medical Sciences, Ministry of Public Health, Thailand

Kasama Sukapirom

Siriraj Hospital

Sansnee Senawong

Siriraj Hospital

\section{Surakameth Mahasirimongkol}

Department of Medical Sciences, Ministry of Public Health

Zheng Quan Toh

Murdoch Children's Research Institute https://orcid.org/0000-0002-0282-5837

Pinklow Umrod

Department of Medical Sciences, Ministry of Public Health, Thailand

Thitiporn Somporn

Department of Medical Sciences, Ministry of Public Health, Thailand

\section{Supaporn Chumpol}

Department of Medical Sciences, Ministry of Public Health, Thailand

Kanokphon Ritthitham

Siriraj Hospital

Kulkanya Chokephaibulkit ( $\sim$ kulkanya.cho@mahidol.ac.th )

Mahidol University

\section{Article}

Keywords: COVID-19, COVID-19 booster vaccination, CoronaVac, ChAdOx1 
Posted Date: December 1st, 2021

DOI: https://doi.org/10.21203/rs.3.rs-1124837/v1

License: (c) (i) This work is licensed under a Creative Commons Attribution 4.0 International License. Read Full License 


\section{Abstract}

The appropriate COVID-19 booster vaccine following inactivated or adenoviral vector COVID-19 vaccination is unclear. We evaluated the safety and immunogenicity of different booster vaccines, inactivated (BBIBP-CorV), chimpanzee adenoviral vector (ChAdOx1), or mRNA (BNT162b2 at full (30 $\mu$ g), or half $(15 \mu \mathrm{g})$ dose $)$ in healthy adults who received 2-dose primary series of either inactivated vaccine (CoronaVac) or ChAdOx1 8-12 weeks earlier. Overall, the adverse events for all booster vaccines were mild and moderate. Two weeks post-booster dose, the neutralising antibody titres against Delta variant in

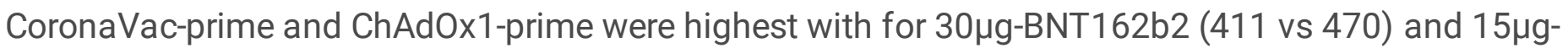
BNT162b2 (499 vs 358); followed by ChAdOx1 (271 vs 69), and BBIBP-CorV (61.3 vs 49). BNT162b2 also induced higher interferon gamma response. Heterologous COVID-19 boosting vaccination with BNT162b2 is the most immunogenic following CoronaVac or ChAdOx1 primary series. A lower dose BNT162b2 may be used as booster in settings with limited vaccine supply.

\section{Introduction}

Both CoronaVac (an inactivated whole-virion SARS-CoV-2 vaccine, Sinovac Life Science) and ChAdOx1 (a chimpanzee adenovirus-vectored vaccine expressing the SARS-CoV-2 spike protein, Oxford, AstraZeneca) are safe and effective against symptomatic COVID-19 caused by the ancestral Wuhan strain, and to a lower extent against the delta variant ${ }^{1-7}$. These two vaccines are among the most widely used vaccines in low- and middle-income countries and some high-income countries.

There are increasing reports of breakthrough infections following COVID-19 vaccination, which is likely due to a combination of waning immunity and the emergence of SARS-CoV-2 variants, particularly the delta variant ${ }^{8-11}$. While the antibody threshold of protection has not been identified, higher antibody levels are likely to be associated with greater protection, including the variants ${ }^{7}$. Furthermore, cell mediated immune responses (CMIR) generated following vaccination are also likely to play an important role in protection.

Several studies have demonstrated improved humoral responses with heterologous COVID-19 primeboost vaccination, primarily on ChAdOx 1 and mRNA vaccines ${ }^{12-16}$. However, other combinations of prime-boost COVID-19 vaccination involving inactivated COVID-19 vaccines have not been evaluated. $A$ recent study of reduced dosage of a booster shot of mRNA-1273 vaccine was found to be highly immunogenic ${ }^{17}$. It is therefore possible that the dosage required for booster may be less than the primary series, particularly with the highly immunogenic vaccine like mRNA vaccines. Reduced dosage of the vaccine will therefore increase accessibility, reduced cost, and could be an important strategy for public health to control the outbreak.

In this study, we examined the immunogenicity and safety of different booster vaccine platforms and reduced dosages (BBIBP-CorV, inactivated vaccine manufactured by Sinopharm; ChAdOx1, chimpanzee adenoviral vector vaccine manufactured by AstraZeneca/Oxford; full dose $(30 \mu \mathrm{g})$ BNT162b2, mRNA 
vaccine manufactured by Pfizer; and half dose $(15 \mu \mathrm{g})$ BNT162b2) in healthy adults aged 18-60 years who received a 2-dose primary series of CoronaVac 4 weeks apart (CoronaVac-prime) or ChAdOx1 vaccine 8-10 weeks apart (ChAd0x1-prime).

\section{Results}

A total of 352 participants were enrolled (179 and 173 participants in CoronaVac- and ChAdOx1-prime group), 285 (81\%) were female, and the median age was 39 (interquartile range, IQR: $31-47$ ) years. The demographic of the study participants receiving different booster vaccine was shown in Table 1.

\section{Adverse events (AEs)}

Among CoronaVac-prime group, the frequency of overall AEs was the highest after boosting with

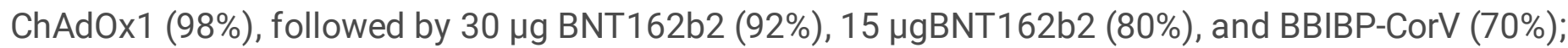
whereas in ChAdOx1-prime group, the highest rate of overall AEs was reported after boosting with $30 \mu \mathrm{g}$ BNT162b2 (98\%), followed by 15 ggBNT162b2 (88\%), ChAdOx1 (72\%), and BBIBP-CorV (61\%) (Fig. 1, Supplementary Table 1). Systemic AEs were in the same trend as local AEs. All AEs were mild (grade 1) or moderate (grade 2) in severity and recovered within 2-3 days. No serious AEs was found in this study.

\section{Anti-SARS-CoV-2 RBD IgG responses}

Prior to the booster dose (8-12 weeks post-primary series), 175/179 (97.8\%) participants in CoronaVacprime and all $(172 / 173,99.4 \%)$ in ChAdOx1-prime were seropositive; however, the anti-RBD IgG geometric mean concentrations (GMCs) were lower in CoronaVac-prime group than ChAdOx1-prime group (36.31 $\mathrm{BAU} / \mathrm{mL}$ range: 33-38 BAU/mL vs 98.27 BAU/mL, range: 90-116 BAU $/ \mathrm{mL}$ ) (Fig. $2 \mathrm{~A})$. For the CoronaVacprime group, at 2 weeks after the booster vaccination, the anti-RBD IgG GMC in the $30 \mu \mathrm{g}$ BNT162b2 group (5,152 BAU/mL) was significantly higher than other booster vaccine groups: $15 \mu \mathrm{g}$ BNT162b2 (3,981 BAU/mL), ChAdOx1 (1,358 BAU/mL), and BBIBP-CorV (154 BAU/mL) (Fig. 2A). A

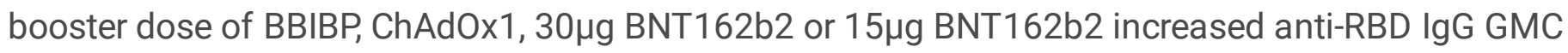
by $4.5,35.6,154.7$, and 105.7 times from baseline, respectively. The geometric mean ratio (GMR, 95\% $\mathrm{Cl})$ between post-boost and post-primary series of CoronaVac were $0.94(0.53,1.67)$ for BBIBP-CorV, 8.26

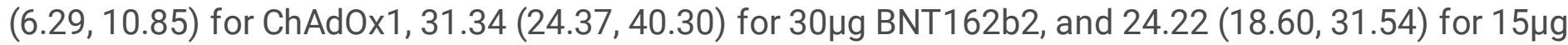
BNT162b2, respectively (Supplementary Table 2).

For the ChAdOx1-prime group, at 2 weeks after the booster dose, the anti-RBG IgG GMC was highest in the

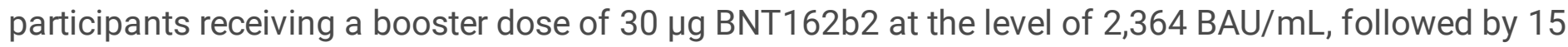
rgBNT162b2 (1,962 BAU/mL), ChAdOx1 (246.4 BAU/mL), and BBIBP-CorV (128.1 BAU/mL). However, there was no statistical difference in the anti-RBD IgG GMC between $30 \mu \mathrm{g}$ and $15 \mu \mathrm{g}$ BNT162b2 groups ( $P=0.1381$ ) (Fig. 2B). A booster dose of BBIBP, ChAdOx1, 30 $\mu$ g BNT162b2, or 15 $\mu$ g BNT162b2 increased GMCs for 1.2, 2.3, 25.1, and 21.8 times from baseline, respectively. The GMR $(95 \% \mathrm{Cl})$ between post-boost and post-primary series of ChAd0x1 were $0.46(0.28,0.65)$ for BBIBP-CorV, $0.88(0.58,1.13)$

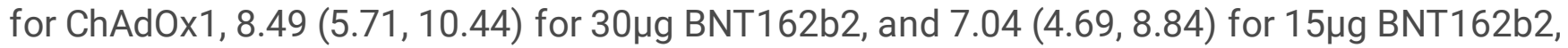


respectively (Supplementary Table 2). The post-boost GMC levels in ChAdOx1-prime group were generally lower than those in the CoronaVac-prime group for all booster vaccines. (Fig. 2C).

\section{Neutralising antibody response against SARS-CoV-2 variants}

The PRNT 50 GMTs against the Delta variant among CoronaVac-prime participants were highest after a booster dose of $15 \mu \mathrm{g}$ BNT162b2 (499.1), followed by $30 \mu \mathrm{g}$ BNT162b2 (411.1), ChAdOx1 (271.2), and BBIBP-CorV (61.3); whereas among ChAdOx1-prime group, the highest PRNT 50 GMT was after a booster dose of $30 \mu \mathrm{g}$ BNT162b2 (470), followed by $15 \mu \mathrm{g}$ BNT162b2 (358.4), ChAdOx1 (69.1), and BBIBP-CorV (49.1) (Fig. 3A). A similar trend was observed for PRNT ${ }_{50}$ GMT against the Beta variant (Fig. 3B). There was no statistical difference of PRNT ${ }_{50}$ GMT between boosting with $30 \mu \mathrm{g}$ and $15 \mu \mathrm{g}$ BNT162b2 regardless of the vaccine given as primary series and the type of variants. The PRNT ${ }_{50} \mathrm{GMT}$ of the Beta variants were around 1.5-fold lower than the Delta variants for both CoronaVac-prime and ChAd0x1-prime groups (Fig. 3C). The GMRs (95\% Cl) of PRNT ${ }_{50}$ titres between post-boost and postprimary series were highest among the participants who received BNT162b2 boosting vaccination in both CoronaVac-prime and ChAd0x1-prime groups for both Delta and Beta variants (Table 2). The anti-SARSCoV-2 RBD IgG levels and the PRNT50 titres against Delta variant (Fig. 4A and Fig. 4B) or Beta variant (Fig. 4C and Fig. 4D) were highly correlated $(r=0.49-0.89)$ particularly in the ChAdOx1-primed group.

\section{Interferon gamma responses (Interferon gamma release assay, IGRA)}

At 8-12 weeks following the primary series, 62/173 (35.8\%) of participants in ChAdOx1-prime group had positive IGRA, while only $45 / 179(25 \%)$ CoronaVac-prime group was positive $(P=0.029)$. Among those who were seronegative in both CoronaVac-prime and ChAdOx1-prime group, IGRA conversion was the highest after a booster dose of $30 \mu \mathrm{g}$ BNT162b2, followed by $15 \mu \mathrm{g}$ BNT162b2, ChAdOx1, and BBIBPCorV (Table 1). Interestingly, none of the study participants who were IGRA-negative at baseline in theChAd0x1-prime group had a positive IGRA response following boosting with BBIBP-CorV or ChAdOx1 (Table 1) (Supplementary Fig. S1).

\section{Discussion}

In this study, we found that BBIBP-CorV, ChAdOx1, BNT162b2 (standard and reduced dosage) given as booster dose to individuals who received either CoronaVac or ChAd0x1 primary series were safe and immunogenic. BNT162b2 as a booster vaccine induced the highest humoral and cellular immunity compared to BBIBP-CorV or ChAdOx1. An interesting observation was the higher immune responses in the CoronaVac-primed group compared to the ChAdOx1-prime group when boosting with BNT162b2 at the similar interval after primary series.

Booster vaccinations using BBIBP-CorV, ChAdOx1, BNT162b2 (standard and reduced dosage) was safe and well tolerated, although the overall proportion who reported AEs (61-98\%) were high, particularly among BNT162b2 recipients, as well as among ChAdOx1 who received it as the first time among 
CoronaVac-prime group. All AEs were mild or moderate in severity and no serious AEs was reported. These AEs rates were similar to those reported in COVID-19 vaccine primary series and booster studies ${ }^{17-}$ 18. The AEs frequencies following ChAdOx 1 and BBIBP-CorV in this study were consistent with those reported in the primary series vaccination in this population ${ }^{7}$.

Heterologous boosting vaccination in our study were found to be more immunogenic than homologous boosting regimen of $\mathrm{ChAdOx} 1$ or homologous vaccine platforms (CoronaVac prime-BBIBP-CorV boost; inactivated vaccines). The higher humoral immune responses following heterologous ChAdOx1 primeBNT162b2 boost vaccination compared to homologous ChAdOx1 prime-ChAd0x1 boost vaccination observed in our study was consistent with what was reported in a cohort study ${ }^{19}$. However, no clinical studies have yet to report on the heterologous boosting responses involving CoronaVac for us to verify our findings. The significant increase in neutralising antibodies against Delta and Beta variants following boosting with BNT162b2 suggests that heterologous boosting with BNT162b2 is likely to be protective against these variants. However, an interesting finding was that individuals in the ChAdOx1-prime group had a blunted neutralising antibody response against the Delta and Beta variants following BBIBP-CorV or ChAdOx1 booster. This suggests that BBIBP-CorV or ChAdOx1 given as a booster to individuals who

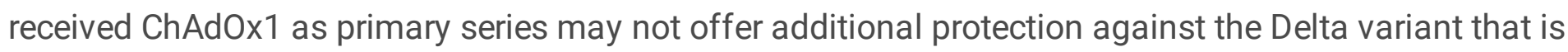
widely circulating globally. However, the number of participants for this analysis was small and larger studies are needed to verify this finding.

While neutralising antibodies are thought to be the primary mechanism of protection ${ }^{7}$, virus-specific memory $T$ cells are also important for protection. Only a third of ChAdOx1-prime and a quarter of CoronaVac-prime participants in our study had positive IGRA 8-12 weeks post primary series, suggesting that CoronaVac induce a poorer $\mathrm{T}$ cell memory response than ChAdOx1. Previous studies evaluating 2dose ChAdOx 1 primary series have reported the generation of robust $\mathrm{T}$ cell responses (measured by interferon gamma ELISPOT) 14 days following the first dose, with no significant increase in $\mathrm{T}$ cell responses following the second dose ${ }^{20-21}$. The discrepancy in $\mathrm{T}$ cell responses from our study could be due to waning immunity, population differences and the different assays used to measure interferon gamma response (Quantiferon vs inteferon gamma ELISPOT). Interestingly, boosting with standard or reduced dosage BNT162b2 induce robust T cell responses, whereas boosting with BBIBP or homologous boosting regimen of ChAdOx1 (third dose) appears to be a poor inducer of $\mathrm{T}$ cell responses. It is important to note that inactivated vaccine (i.e. BBIBP) may have other antigens (i.e. $\mathrm{M}$ or $\mathrm{N}$ proteins) that induce $T$ cell responses ${ }^{22}$, whereas in our study, we only examined $T$ cell responses to $S$ protein, and thus may have underestimated the cellular responses. The low $T$ cell boosting responses following homologous boosting regimen of ChAdOx1 is in line with the low neutralising antibody boosting responses against the Delta and Beta variants observed in this study. This could be explained by the antivector interference because of the primary vaccination, although more studies are needed to confirm this finding. 
Our findings in both vaccine-prime groups suggested that less amount of antigen may be sufficient for boosting. We found that half-dose of $15 \mu \mathrm{g}$ BNT162b2 was equally immunogenic as the standard dose of $30 \mu \mathrm{g}$, but with less reactogenicity. This is in concordance with a study on mRNA1273 vaccine where a booster dose of mRNA1273 vaccine at half the normal dosage $(50 \mu \mathrm{g})$ was able to induce significantly higher neutralising antibodies than the level induced following primary series and against the VOCs ${ }^{17}$. Taken together, a lower mRNA vaccine dose may be an option for COVID-19 booster vaccination given the limited vaccine supply. However, the persistence of immunity and the efficacy following reduced dosages in booster vaccination remains to be determined.

There are some limitations in this study. First, our study was conducted in an open label manner due to the availability of each vaccine at a different timing. While this may lead to selection bias and subjective outcomes such as AEs, the demographic characteristics of the participants in the booster groups were very similar, suggesting that this is unlikely to influence our findings. Second, our sample size is small, particularly those who received BBIBP as booster, and therefore, the data need to be interpreted with caution. Furthermore, it is unclear if similar findings will be found if the duration between the last dose of primary series and the booster dose is $>12$ weeks (8-12 weeks in this study). Last, the participants in this study were healthy adults therefore results may not be generalisable to other populations such as elderly or those with underlying diseases.

In conclusion, we found that a booster dose of BNT162b2 given to individuals previously vaccinated with CoronaVac or ChaAdOx1 primary series is the most immunogenic. Our study findings have important implications on the choice of booster dose following CoronaVac or ChAdOx1 primary series, and are most relevant for countries that have introduced these vaccines to date. Furthermore, our study indicates that reduced dosage of BNT162b2 may be used as a booster dose instead of the standard dosage. This will aid in improving global access to COVID-19 vaccines.

\section{Methods}

\section{Study design and participants}

This single-center prospective cohort study enrolled 352 healthy adults aged 18-60 years of age at Siriraj Hospital, a university-based referral center in Bangkok, Thailand, from March to September 2021. The eligible participants were those who have received either 2-dose 4 weeks apart of CoronaVac or 8-10 weeks apart of ChAd0x1 primary series vaccination in the period of 8-12 weeks prior the screening day. The exclusion criteria were history of SARS-CoV-2 infection; received prior administration of current prophylactic treatment or investigational agents against COVID-19 within 90 days; had an unstable underlying disease; history of vaccine anaphylaxis; being pregnant; immunocompromised or currently receiving immunosuppressive agents. The study procedures, risks, and benefits were explained to all participants before obtaining written informed consent. The study protocol was approved by the Siriraj Institutional Review Board (COA no. Si 537/2021). The procedures in this study adhere to the tenets of the Declaration of Helsinki. 


\section{Study Procedures}

The participants with prior 2-dose primary series of CoronaVac (CoronaVac-prime) or ChAd0x1 (ChAdOx1-prime) were openly assigned to receive one of the four intramuscular booster vaccinations: BBIBP-CorV (manufactured by Sinopharm), ChAd0x1, full dose (30 $\mu \mathrm{g})$ BNT162b2 (manufactured by Pfizer), and half dose $(15 \mu \mathrm{g})$ BNT162b2. Prior to enrollment, the potential participants who were interested to participate were provided an on-line questionnaire to select their first and second choice of booster vaccine which will be available at the time. The assigned booster vaccine was matched with the indicated pre-enrollment choice and the availability of the vaccine on the study day.

The participants were observed for at least 30 min following vaccination for any immediate adverse events (AE). Participants were instructed to record self-assessment signs or symptoms in an electronic diary (eDiary) for seven days after vaccination to track AE including solicited local and systemic adverse reactions. An AE in this study will be defined as any unfavorable or unexpected sign, symptom, or disease (including an unexpected finding in a laboratory evaluation) that occurs in any participant whether it is related to the vaccination product. Solicited local AE included pain, erythema, and swelling/induration at the injection site, and localized axillary swelling or tenderness ipsilateral to the injection arm. Solicited systemic AE were headache, fatigue, myalgia, arthralgia, nausea, vomiting, rash, fever, and chills. The severity of solicited AE were graded using a numerical scale from 1 to 4 based on two guides; Toxicity Grading Scale for Healthy Adult and Adolescent Volunteers Enrolled in Preventive Vaccine Clinical Trials from the United States Food and Drug Administration (FDA), and in the Common Terminology Criteria for Adverse Events - Version 5.0 guide by the United States National Cancer Institute $(\mathrm{NCl} / \mathrm{NIH})$.

Blood samples were collected at baseline (pre-booster), and two weeks after booster vaccination (postboost) to determine the level of antibody response (IgG) against receptor binding domain (RBD) of the SARS-CoV-2 S1 subunit spike protein (anti-RBD IgG) and 50\% plaque reduction neutralisation test (PRNT ${ }_{50}$ ) against Delta (B1.1617.2) and Beta (B.1.351) variants. The whole blood samples were collected for cellular immunity testing by the QuantiFERON SARS-CoV-2 interferon gamma release assay (IGRA) at baseline. The participants who had IGRA levels below the cut-off point were tested again at 2 weeks following the booster vaccination.

\section{Chemiluminescent microparticle assay (CMIA) for anti-SARS-CoV-2 RBD IgG}

The plasma samples were stored at $-80^{\circ} \mathrm{C}$ until testing in batch. The anti-RBD IgG was quantitatively measured by CMIA using the SARS-CoV-2 IgG II Quant (Abbott, List No. 06S60) on the ARCHITECT i System. This assay linearly measures the level of antibody between $21.0-40,000.0$ arbitrary unit $(\mathrm{AU}) / \mathrm{mL}$, which was converted later to $\mathrm{WHO}$ international standard concentration as binding antibody unit per $\mathrm{mL}(\mathrm{BAU} / \mathrm{mL})$ following the equation provided by the manufacturer $(\mathrm{BAU} / \mathrm{mL}$ $=0.142 \times \mathrm{AU} / \mathrm{mL}$ ). The level greater or equal to the cutoff value of $50 \mathrm{AU} / \mathrm{mL}$ or $7.1 \mathrm{BAU} / \mathrm{mL}$ was defined 
as seropositive. Seroconversion was determined as becoming seropositive in those who had seronegative at baseline or increase of 4 -fold titres above the baseline seropositive levels.

$50 \%$ plaque reduction neutralisation test (PRNT 50$)$

The standard live virus $50 \%$ plaque reduction neutralisation test $\left(\mathrm{PRNT}_{50}\right.$ ) against Delta variant (B.1.617.2) and Beta variant (B.1.351) were performed. Vero cells were seeded at $2 \times 10^{5}$ cells $/$ well/ $3 \mathrm{ml}$ and placed in $37^{\circ} \mathrm{C}, 5 \% \mathrm{CO}_{2}$ incubator for 1 day. Test sera were initially diluted at 1:10, 1:40, 1:160 and $1: 640$, respectively. SARS-CoV-2 virus was diluted in culture medium to yield 40-120 plaques/well in the virus control wells. Cell control wells, convalescent patient serum and normal human serum were also included as assay controls. The neutralisation was performed by mixing the equal volume of diluted serum and the optimal plaque numbers of SARS CoV-2 virus at $37^{\circ} \mathrm{C}$ in water bath for $1 \mathrm{~h}$. After removing the culture medium from Vero cell culture plates, 200 ul of the virus-serum antibody mixture were inoculated into monolayer cells and then rocked the culture plates every $15 \mathrm{~min}$ for $1 \mathrm{~h}$. Three $\mathrm{ml}$ of overlay semisolid medium (containing $1 \%$ of carboxymethylcellulose, Sigma Aldrich, USA, with $1 \%$ of 10,000 units/ml penicillin, 10,000 ug/ml streptomycin (Sigma, USA), and 10\% FBS) were replaced after removing excessive viruses. All plates were incubated at $37^{\circ} \mathrm{C}, 5 \% \mathrm{CO}_{2}$ for 7 days. Cells were fixed with $10 \%(\mathrm{v} / \mathrm{v})$ formaldehyde then stained with $0.5 \%$ crystal violet in PBS. The number of plaques formed was counted in triplicate wells and percentage of plaque reduction at $50 \%\left(\mathrm{PRNT}_{50}\right)$ was calculated. The $\mathrm{PRNT}_{50}$ titre of test sample is defined as the reciprocal of the highest test serum dilution for which the virus infectivity is reduced by $50 \%$ when compared with the average plaque counts of the virus control and was calculated by using a four-point linear regression method. Plaque counts for all serial dilutions of serum were scored to ensure that there was a dose response.

QuantiFERON SARS-CoV-2 interferon gamma release assay (IGRA)

SARS-CoV-2 specific T cell responses were assessed by whole blood IGRA using QIAGEN's proprietary mixes of SARS-CoV-2 S protein following manufacture's instruction. Briefly, whole blood samples were collected into the unstimulated tube as negative control and stimulated tubes containing SARS-CoV-2 S peptide designed for $\mathrm{CD}^{+} \mathrm{T}$ cell (Ag.1), for $\mathrm{CD} 8^{+} \mathrm{T}$ cells (Ag.2) and for mitogen as positive control. The specimen was incubated at $37 \otimes C$ for 16-24 hours and centrifuged to separate plasma. Interferon-gamma (IFN-Y) concentration in the plasma fraction was measured with an automated QuantiFERON SARS-CoV2 ELISA instrument for research use only (RUO) and reported in International Units per $\mathrm{mL}(\mathrm{IU} / \mathrm{mL})^{23-24}$. The cut-off for positivity was determined as the level above the mean plus three standard deviations of the negative control. Using 61 negative controls at the study site, the cut-offs for Ag1 and Ag2 were $>0.12$ $\mathrm{IU} / \mathrm{mL}$ and $>0.17 \mathrm{IU} / \mathrm{mL}$, respectively. A positive response to either of the two peptides pools was considered positive.

\section{Statistical Analysis}


The AEs endpoints were presented as frequencies and Chi-square test was used to test for statistical difference. The immunological endpoints including anti-SARS-CoV-2 RBD IgG concentration and PRNT 50 titres at 2 weeks after booster vaccination (post-boost) were reported as geometric mean concentration (GMC) with 95\% confidence interval (Cl) if the data was positively skewed. The PRNT 50 titres below 10 were arbitrarily assigned a value of 5 . We used the anti-RBD IgG GMC and the PRNT 50 geometric mean titres (GMT) at two weeks after the second dose of primary series vaccination (post-primary series) from our previous study as the references for comparison. The post-primary anti-RBD IgG GMC levels was 164.4 BAU/mL and 278.5 BAU/mL in 2-dose CoronaVac and ChAdOx1, respectively, while the respective PRNT $_{50}$ GMT was 21.2 and 69.7 for Delta variant and 10.2 and 43.5 for Beta variant ${ }^{8}$. Of note, 278 (79\%) subjects in this study also participated in the primary series study with the data included. The geometric mean ratio (GMR) with $95 \% \mathrm{Cl}$ were analyzed between the post-boosting levels/titres and post-primary series levels/titres references. Unpaired t-test was used to compare GMC of the IgG concentrations between groups using GraphPad Prism 9 version 9.2.0 (283) (GraphPad Software, CA, USA). A p-value less than 0.05 is considered statistically significance. All statistical analyses were conducted using STATA version 17 (Stata Corp, LP, College Station, TX, USA), SAS Studio.

\section{Declarations}

\section{Data Availability}

The datasets generated during and/or analyzed during the current study are available from the corresponding author on reasonable request.

\section{Acknowledgement}

The authors gratefully acknowledge the Siriraj Institute of Clinical Research (SICRES) team, Abbott Laboratories Ltd. for technical supports and all health care workers who took part and enabled this study to be possible.

\section{Author Contributions}

N.A. and S.N. equally contributed to the research work. Conceptualization and Methodology, N.A., S.N., J.S., and K.C.; Formal analysis and data curation: N.A., J.S., S.N. R.T; Project administration, N.A, J.S., S.N.; Supervision, K.C.; Resources and Funding, K.C. All involved with investigation, and writingreview and editing.

\section{Conflict of Interest Declaration}

All authors declare no personal or professional conflicts of interest, and no financial support from the companies that produce and/or distribute the drugs, devices, or materials described in this report.

\section{Funding Disclosure}


This study was supported by the National Research Council of Thailand. The Abbott Laboratories

Ltd. partially supported the reagents for the anti-SARS-CoV-2 RBD IgG in this study.

\section{References}

1. Tanriover, M. et al. Efficacy and safety of an inactivated whole-virion SARS-CoV-2 vaccine (CoronaVac): interim results of a double-blind, randomised, placebo-controlled, phase 3 trial in Turkey. The Lancet 398, 213-222 (2021).

2. Voysey, M. et al. Safety and efficacy of the ChAdOx1 nCoV-19 vaccine (AZD1222) against SARS-CoV2: an interim analysis of four randomised controlled trials in Brazil, South Africa, and the UK. The Lancet 397, 99-111 (2021).

3. Jara, A. et al. Effectiveness of an Inactivated SARS-CoV-2 Vaccine in Chile. New England Journal of Medicine 385, 875-884 (2021).

4. Lopez Bernal, J.et al.Effectiveness of Covid-19 Vaccines against the B.1.617.2 (Delta) Variant. New England Journal of Medicine 385, 585-594 (2021).

5. Li, X. et al. Effectiveness of inactivated SARS-CoV-2 vaccines against the Delta variant infection in Guangzhou: a test-negative case-control real-world study. Emerging Microbes \& Infections 10, 1751-1759 (2021).

6. Mallapaty, S. China's Covid vaccines have been crucial - now immunity is waning. Nature News (2021). Available at: https://www.nature.com/articles/d41586-021-02796-w. (Accessed: 21st October 2021)

7. Angkasekwinai, N.et al.Safety and Immunogenicity of CoronaVac and ChAdOx1 Against the SARSCoV-2 Circulating Variants of Concern (Alpha, Delta, Beta) in Thai Healthcare Workers.Preprint at medRxiv https://doi:10.1101/2021.10.03.21264451 (2021).

8. Khoury, D.et al.Neutralizing antibody levels are highly predictive of immune protection from symptomatic SARS-CoV-2 infection. Nature Medicine 27, 1205-1211 (2021).

9. Naaber, P.et al.Dynamics of antibody response to BNT162b2 vaccine after six months: a longitudinal prospective study. The Lancet Regional Health <background-color:\#CCFF99;idirection:rt|;></background-color:\#CCFF99;idirection:rtl;> Europe 10, 100208 (2021).

10. Lopez Bernal, J.et al.Effectiveness of the Pfizer-BioNTech and Oxford-AstraZeneca vaccines on covid-19 related symptoms, hospital admissions, and mortality in older adults in England: test negative case-control study. British Medical Journal 373,n1088(2021).

11. AlQahtani, M.et al.Morbidity and mortality from COVID-19 post-vaccination breakthrough infections in association with vaccines and the emergence of variants in Bahrain.Preprint at Research Square https://doi.org/10.21203/rs.3.rs-828021/v1 (2021).

12. Borobia, A.et al.Immunogenicity and reactogenicity of BNT162b2 booster in ChAdOx1-S-primed participants (CombiVacS): a multicentre, open-label, randomised, controlled, phase 2 trial. The Lancet 398, 121-130 (2021). 
13. Barros-Martins, J.et al.Humoral and cellular immune response against SARS-CoV-2 variants following heterologous and homologous ChAdOx1 nCoV-19/BNT162b2 vaccination.Preprint at medRxiv https://doi:10.1101/2021.06.01.21258172 (2021).

14. Gross, R.et al.Heterologous ChAdOx1 nCoV-19 and BNT162b2 prime-boost vaccination elicits potent neutralizing antibody responses and T cell reactivity.Preprint at medRxiv https://doi:10.1101/2021.05.30.21257971 (2021).

15. Yorsaeng, R.et al.Immune response elicited from heterologous SARS-CoV-2 vaccination: Sinovac (CoronaVac) followed by AstraZeneca (Vaxzevria).Preprint at medRxiv https://doi:10.1101/2021.09.01.21262955 (2021).

16. Nordström, P., Ballin, M. \& Nordström, A. Effectiveness of heterologous ChAdOx1 nCoV-19 and mRNA prime-boost vaccination against symptomatic Covid-19 infection in Sweden: A nationwide cohort study. The Lancet Regional Health - Europe 100249 (2021).

17. Choi, A.et al.Safety and immunogenicity of SARS-CoV-2 variant mRNA vaccine boosters in healthy adults: an interim analysis. Nature Medicine (2021).

18. Menni, C.et al.Vaccine side-effects and SARS-CoV-2 infection after vaccination in users of the COVID Symptom Study app in the UK: a prospective observational study. The Lancet Infectious Diseases 21, 939-949 (2021).

19. Barros-Martins, J. et al. Immune responses against SARS-CoV-2 variants after heterologous and homologous ChAdOx1 nCoV-19/BNT162b2 vaccination. Nature Medicine 27, 1525-1529 (2021).

20. Folegatti, P. et al. Safety and immunogenicity of the ChAdOx $1 \mathrm{nCoV}-19$ vaccine against SARS-CoV-2: a preliminary report of a phase $1 / 2$, single-blind, randomised controlled trial. The Lancet 396, 467478 (2020).

21. Ramasamy, M. et al. Safety and immunogenicity of ChAdOx $1 \mathrm{nCoV}-19$ vaccine administered in a prime-boost regimen in young and old adults (COV002): a single-blind, randomised, controlled, phase 2/3 trial. The Lancet 396, 1979-1993 (2020).

22. Vályi-Nagy, I. et al. Comparison of antibody and T cell responses elicited by BBIBP-CorV (Sinopharm) and BNT162b2 (Pfizer-BioNTech) vaccines against SARS-CoV-2 in healthy adult humans. GeroScience 43, 2321-2331 (2021).

23. Murugesan, K.et al.Interferon- $\gamma$ Release Assay for Accurate Detection of Severe Acute Respiratory Syndrome Coronavirus 2 T-Cell Response. Clinical Infectious Diseases 73, e3130-e3132 (2020).

24. Martínez-Gallo, M.et al.Commercialized kit to assess T-cell responses against SARS-CoV-2 $S$ peptides. A pilot study in Health Care Workers.Preprint at medRxiv https://doi:10.1101/2021.03.31.21254472 (2021).

\section{Tables}

Table 1 Characteristics of 352 participants classified by primary series vaccination. 


\begin{tabular}{|c|c|c|c|c|c|}
\hline Demographics & Type of bo & ster vaccinati & & & \\
\hline CoronaVac-prime $(\mathrm{n}=179)$ & & & & & \\
\hline & $\begin{array}{l}\text { BBIBP- } \\
\text { CorV } \\
n=14\end{array}$ & $\begin{array}{l}\text { ChAdOx1 } \\
n=65\end{array}$ & $\begin{array}{l}30 \mu \mathrm{g} \\
\text { BNT162b2 } \\
\mathrm{n}=50\end{array}$ & $\begin{array}{l}15 \mu \mathrm{g} \\
\text { BNT162b2 } \\
\mathrm{n}=50\end{array}$ & $p$-value \\
\hline Age (years), median (IQR) & $\begin{array}{l}31 \\
(27,41.5)\end{array}$ & $\begin{array}{l}36.6 \\
(29.5,44)\end{array}$ & $\begin{array}{l}32 \\
(28,41.8)\end{array}$ & $\begin{array}{l}40 \\
(31.5,45.3)\end{array}$ & 0.018 \\
\hline Female, n (\%) & $12(85.7)$ & $51(78.5)$ & $40(80.0)$ & $33(66.0)$ & 0.249 \\
\hline $\mathrm{BMI}\left(\mathrm{kg} / \mathrm{m}^{2}\right)$, median (IQR) & $\begin{array}{l}25.2 \\
(21.1 \\
31.6)\end{array}$ & $\begin{array}{l}23.4 \\
(20.9,27.1)\end{array}$ & $\begin{array}{l}22.1 \\
(19.5,25.5)\end{array}$ & $\begin{array}{l}23.9 \\
(20.9,26.0)\end{array}$ & 0.325 \\
\hline $\begin{array}{l}\text { Anti-RBD IgG GMC at baseline } \\
(95 \% \mathrm{Cl})\end{array}$ & $\begin{array}{l}34.32 \\
(22.33 \\
52.77)\end{array}$ & $\begin{array}{l}38.18 \\
(31.21 \\
46.71)\end{array}$ & $\begin{array}{l}33.31 \\
(26.72, \\
41.53)\end{array}$ & $\begin{array}{l}37.67 \\
(31.65, \\
44.84)\end{array}$ & 0.7616 \\
\hline $\begin{array}{l}\text { Anti-RBD IgG GMC after } \\
\text { boosting }(95 \% \mathrm{Cl})\end{array}$ & $\begin{array}{l}154.6 \\
(92.11 \\
259.47)\end{array}$ & $\begin{array}{l}1358.0 \\
(1141.84 \\
1615.07)\end{array}$ & $\begin{array}{l}5152.2 \\
(4491.65 \\
5909.83)\end{array}$ & $\begin{array}{l}3981.1 \\
(3397.15 \\
4665.42)\end{array}$ & $<0.0001$ \\
\hline $\begin{array}{l}\text { Baseline SARS-CoV-2 IGRA } \\
\text { positive, } n(\%)\end{array}$ & $5(35.7)$ & $12(18.5)$ & $16(32)$ & $12(24)$ & 0.301 \\
\hline $\begin{array}{l}\text { Post-boosting IGRA positive } \\
\text { among baseline negative } \\
\text { participants, } n(\%)\end{array}$ & $\begin{array}{l}1 / 9 \\
(11.1)\end{array}$ & $\begin{array}{l}26 / 53 \\
(49.1)\end{array}$ & $\begin{array}{l}28 / 34 \\
(82.4)\end{array}$ & $\begin{array}{l}30 / 38 \\
(79.0)\end{array}$ & $<0.0001$ \\
\hline ChAdOx1-prime $(n=173)$ & & & & & \\
\hline & $\begin{array}{l}\text { BBIBP- } \\
\text { CorV } \\
n=23\end{array}$ & $\begin{array}{l}\text { ChAdOx1 } \\
\mathrm{n}=50\end{array}$ & $\begin{array}{l}30 \mu \mathrm{g} \\
\text { BNT162b2 } \\
\mathrm{n}=50\end{array}$ & $\begin{array}{l}15 \mu \mathrm{g} \\
\text { BNT162b2 } \\
\mathrm{n}=50\end{array}$ & $p$-value \\
\hline Age (years), median (IQR) & $\begin{array}{l}51 \\
(42,59)\end{array}$ & $\begin{array}{l}45.5 \\
(36,57)\end{array}$ & $\begin{array}{l}34 \\
(30,43)\end{array}$ & $\begin{array}{l}41.5 \\
(34,49.5)\end{array}$ & 0.001 \\
\hline Female, n (\%) & $21(91.3)$ & $47(94.0)$ & $37(74.0)$ & $44(88.0)$ & 0.001 \\
\hline BMI $\left(\mathrm{kg} / \mathrm{m}^{2}\right)$, median (IQR) & $\begin{array}{l}24.8 \\
(22.4 \\
27.6)\end{array}$ & $\begin{array}{l}23.8 \\
(21.3,26.7)\end{array}$ & $\begin{array}{l}21.4 \\
(19.3,24.7)\end{array}$ & $\begin{array}{l}23.3 \\
(20.4,26.5)\end{array}$ & 0.001 \\
\hline Anti-RBD IgG at baseline & 106.6 & 105.7 & 95.98 & 90.11 & 0.7661 \\
\hline
\end{tabular}




\begin{tabular}{|c|c|c|c|c|c|}
\hline$(95 \% \mathrm{Cl})$ & $\begin{array}{l}(70.89 \\
160.29)\end{array}$ & $\begin{array}{l}(80.97 \\
137.97)\end{array}$ & $\begin{array}{l}(75.84 \\
121.45)\end{array}$ & $\begin{array}{l}(73.62 \\
110.30)\end{array}$ & \\
\hline $\begin{array}{l}\text { Anti-RBD IgG GMC after } \\
\text { boosting }(95 \% \mathrm{Cl})\end{array}$ & $\begin{array}{l}128.1 \\
(93.52 \\
175.37)\end{array}$ & $\begin{array}{l}246.4 \\
(199.59 \\
304.20)\end{array}$ & $\begin{array}{l}2363.8 \\
(2005.58 \\
2786.06)\end{array}$ & $\begin{array}{l}1961.9 \\
(1624.61 \\
2369.10)\end{array}$ & $<0.0001$ \\
\hline $\begin{array}{l}\text { Baseline SARS-CoV-2 IGRA } \\
\text { positive, n (\%) }\end{array}$ & $13(56.5)$ & $26(52.0)$ & 9 (18.0) & $14(28.0)$ & $<0.0001$ \\
\hline $\begin{array}{l}\text { Post-boosting IGRA positive } \\
\text { among baseline negative } \\
\text { participants, } n(\%)\end{array}$ & $0 / 10$ & $0 / 24$ & $\begin{array}{l}31 / 41 \\
(75.6)\end{array}$ & $\begin{array}{l}24 / 26 \\
(66.7)\end{array}$ & $<0.0001$ \\
\hline
\end{tabular}

GMC: geometric mean concentration; IGRA: QuantiFERON SARS-CoV-2 interferon gamma release assay

Table 2 The 50\% plaque reduction neutralization ( $\mathrm{PRNT}_{50}$ ) geometric mean antibody titers (GMT) and the geometric mean ratio (GMR) between post-boosting and post-primary series*. 


\begin{tabular}{|c|c|c|c|c|c|}
\hline \multirow{2}{*}{$\begin{array}{l}\text { PRNT }_{50} \text { GMT } \\
\text { CoronaVac-prime }(n=104)\end{array}$} & \multicolumn{5}{|c|}{ Type of booster vaccinations } \\
\hline & & & & & \\
\hline & BBIBP- & \multirow{3}{*}{$\begin{array}{l}\text { ChAdOx } 1 \\
\mathrm{n}=30\end{array}$} & \multirow{3}{*}{$\begin{array}{l}30 \mu \mathrm{g} \\
\text { BNT162b2 } \\
\mathrm{n}=30\end{array}$} & \multirow{3}{*}{$\begin{array}{l}15 \mu \mathrm{g} \\
\text { BNT162b2 } \\
\mathrm{n}=30\end{array}$} & \multirow[t]{3}{*}{$p$-value } \\
\hline & & & & & \\
\hline & $\mathrm{n}=14$ & & & & \\
\hline \multirow{2}{*}{$\begin{array}{l}\mathrm{GMT}(95 \% \mathrm{Cl}) \text { against Delta } \\
\text { variant }\end{array}$} & 61.3 & \multirow{2}{*}{$\begin{array}{l}271.2 \\
(222.54 \\
330.49)\end{array}$} & \multirow{2}{*}{$\begin{array}{l}411.1 \\
(311.71,542.16)\end{array}$} & \multirow{2}{*}{$\begin{array}{l}499.12 \\
(418.54 \\
595.21)\end{array}$} & \multirow[t]{2}{*}{$<0.0001$} \\
\hline & $\begin{array}{l}\text { (35.07) } \\
107.02)\end{array}$ & & & & \\
\hline \multirow{2}{*}{$\begin{array}{l}\text { GMR }(95 \% \mathrm{Cl}) \text { between post- } \\
\text { boosting and post-primary } \\
\text { series* against Delta variant }\end{array}$} & 2.89 & 12.79 & 19.39 & 23.54 & \multirow[t]{2}{*}{$<0.0001$} \\
\hline & $\begin{array}{l}(1.52 \\
5.50)\end{array}$ & $\begin{array}{l}(9.06 \\
18.06)\end{array}$ & $(13.04,28.84)$ & $\begin{array}{l}(16.89 \\
32.82)\end{array}$ & \\
\hline $\begin{array}{l}\mathrm{GMT}(95 \% \mathrm{Cl}) \text { against Beta } \\
\text { variant }\end{array}$ & $\begin{array}{l}37.2 \\
(18.00 \\
76.91)\end{array}$ & $\begin{array}{l}170.5 \\
(124.65 \\
233.13)\end{array}$ & $\begin{array}{l}306.7(221.44 \\
424.71)\end{array}$ & $\begin{array}{l}322.8 \\
(239.34 \\
435.25)\end{array}$ & $<0.0001$ \\
\hline \multirow{2}{*}{$\begin{array}{l}\text { GMR }(95 \% \mathrm{Cl}) \text { between post- } \\
\text { boosting and post-primary } \\
\text { series* against Beta variant }\end{array}$} & 3.65 & 16.72 & 30.07 & 31.65 & \multirow[t]{2}{*}{$<0.0001$} \\
\hline & $\begin{array}{l}(1.65 \\
8.08)\end{array}$ & $\begin{array}{l}(11.11 \\
25.15)\end{array}$ & $(19.79,45.69)$ & $\begin{array}{l}(21.27 \\
47.09)\end{array}$ & \\
\hline \multicolumn{6}{|l|}{ ChAd0x1-prime } \\
\hline & $\begin{array}{l}\text { BBIBP- } \\
\text { CorV }\end{array}$ & \multirow{3}{*}{$\begin{array}{l}\text { ChAdOx } 1 \\
\mathrm{n}=30\end{array}$} & \multirow{3}{*}{$\begin{array}{l}30 \mu \mathrm{g} \\
\text { BNT162b2 } \\
\mathrm{n}=30\end{array}$} & $15 \mu \mathrm{g}$ & \multirow[t]{3}{*}{$p$-value } \\
\hline & & & & BNT162b2 & \\
\hline & $11-\angle 2$ & & & $n=30$ & \\
\hline $\begin{array}{l}\mathrm{GMT}(95 \% \mathrm{Cl}) \text { against Delta } \\
\text { variant }\end{array}$ & $\begin{array}{l}49.0 \\
(37.56 \\
64.05)\end{array}$ & $\begin{array}{l}69.1 \\
(50.14 \\
95.14)\end{array}$ & $\begin{array}{l}470.1(395.49 \\
558.89)\end{array}$ & $\begin{array}{l}358.4 \\
(276.13 \\
465.26)\end{array}$ & $<0.0001$ \\
\hline \multirow{2}{*}{$\begin{array}{l}\text { GMR between post-boosting } \\
\text { and post-primary series* }(95 \% \\
\mathrm{Cl}) \text { against Delta variant }\end{array}$} & 0.70 & 0.99 & \multirow{2}{*}{$\begin{array}{l}6.74(4.45 \\
10.23)\end{array}$} & 5.14 & \multirow[t]{2}{*}{$<0.0001$} \\
\hline & $\begin{array}{l}(0.44 \\
1.12)\end{array}$ & $\begin{array}{l}(0.60 \\
1.63)\end{array}$ & & $\begin{array}{l}(3.24 \\
8.16)\end{array}$ & \\
\hline $\begin{array}{l}\mathrm{GMT}(95 \% \mathrm{Cl}) \text { against Beta } \\
\text { variant }\end{array}$ & $\begin{array}{l}28.1 \\
(18.08 \\
43.53)\end{array}$ & $\begin{array}{l}38.2 \\
(26.06 \\
56.05)\end{array}$ & $\begin{array}{l}292.9(233.73 \\
367.17)\end{array}$ & $\begin{array}{l}250.0 \\
(182.95 \\
341.51)\end{array}$ & $<0.0001$ \\
\hline \multirow{2}{*}{$\begin{array}{l}\text { GMR }(95 \% \mathrm{Cl}) \text { between post- } \\
\text { boosting and post-primary } \\
\text { series* against Beta variant }\end{array}$} & \multirow[t]{2}{*}{0.65} & \multirow[t]{2}{*}{0.88} & 6.73 & \multirow[t]{2}{*}{5.75} & \multirow[t]{2}{*}{$<0.0001$} \\
\hline & & & $(4.42,10.26)$ & & \\
\hline
\end{tabular}


*The post primary series GMC was derived from the study in the same setting as the current study (Reference: Angkasekwinai, N. et al. Safety and Immunogenicity of CoronaVac and ChAdOx 1 Against the SARS-CoV-2 Circulating Variants of Concern (Alpha, Delta, Beta) in Thai Healthcare Workers. Preprint at medRxiv https://doi:10.1101/2021.10.03.21264451 (2021)). The post primary series GMT (95\% $\mathrm{Cl})$ at 2 weeks after the second dose of the 2-dose homologous CoronaVac, 4 weeks apart, were 21.2 (16.07, 27.87) and $10.2(7.92,13.12)$ against Delta and Beta variants, respectively; and after 2-dose homologous ChAdOx1,10 weeks apart, were $69.7(48.08,101.00)$ and $43.5(30.73,61.72)$ against Delta and Beta variants, respectively.

\section{Supplementary Tables}

Supplementary Table 1 Adverse events of following booster vaccination. 


\begin{tabular}{|c|c|c|c|c|c|}
\hline Adverse events (AEs) & BBIBP-CorV & ChAdOx1 & $30 \mu \mathrm{g}$ & $15 \mu \mathrm{g}$ & $\mathrm{p}$-value \\
\hline & & & BNT162b2 & BNT162b2 & \\
\hline CoronaVac-prime $(n=179)$ & $n=14$ & $\mathrm{n}=65$ & $n=50$ & $n=50$ & \\
\hline Overall AEs (\%) & $10(71.43)$ & $64(98.46)$ & $46(92.0)$ & $40(80.0)$ & 0.002 \\
\hline Injection site reaction (\%) & $9(64.29)$ & $62(95.38)$ & $46(92.00)$ & $36(72.00)$ & $<0.001$ \\
\hline Fatigue (\%) & $2(14.29)$ & $46(70.77)$ & $26(52.0)$ & $10(20.00)$ & $<0.001$ \\
\hline Headache (\%) & $1(7.14)$ & $12(18.46)$ & $25(50.0)$ & $18(36.0)$ & $<0.001$ \\
\hline Myalgia (\%) & $6(42.86)$ & $54(83.08)$ & $2(4.0)$ & $1(2.0)$ & $<0.001$ \\
\hline Malaise (\%) & 0 & $1(1.54)$ & $31(62.0)$ & $20(40.0)$ & $<0.001$ \\
\hline Nausea (\%) & $2(14.29)$ & $20(30.77)$ & $7(14.0)$ & $5(10.0)$ & 0.119 \\
\hline Diarrhea (\%) & $1(7.14)$ & $12(18.46)$ & $3(6.0)$ & $6(12.0)$ & 0.284 \\
\hline Fever (\%) & $1(7.14)$ & $25(38.46)$ & $4(8.0)$ & $1(2.0)$ & $<0.001$ \\
\hline Rash (\%) & $2(14.29)$ & $9(13.85)$ & $7(14.0)$ & $2(4.0)$ & 0.341 \\
\hline Somnolence (\%) & 0 & 0 & $2(4.0)$ & $2(4.0)$ & 0.357 \\
\hline Flu-like symptoms (\%) & 0 & $4(6.15)$ & $1(2.0)$ & $1(2.0)$ & 0.453 \\
\hline Arthralgia (\%) & 0 & $2(3.08)$ & $1(2.0)$ & $1(2.0)$ & 0.906 \\
\hline Dizziness (\%) & 0 & $1(1.54)$ & $1(2.0)$ & 0 & 0.758 \\
\hline Paresthesia (\%) & $2(14.29)$ & $1(1.54)$ & $1(2.0)$ & 0 & 0.014 \\
\hline Vomiting (\%) & 0 & $4(6.15)$ & 0 & $1(2.0)$ & 0.199 \\
\hline ChAdOx1-prime $(n=173)$ & $n=23$ & $n=50$ & $n=50$ & $n=50$ & \\
\hline Overall AEs (\%) & $14(60.87)$ & $36(72.0)$ & $49(958.0)$ & $44(89.0)$ & $<0.001$ \\
\hline Injection site reaction (\%) & $9(39.13)$ & $28(56.0)$ & $47(94.00)$ & $44(88.00)$ & $<0.001$ \\
\hline Fatigue (\%) & $3(13.04)$ & $18(36.0)$ & $34(68.0)$ & $20(40.00)$ & $<0.001$ \\
\hline Headache (\%) & $6(26.09)$ & $15(30.0)$ & $28(56.0)$ & $28(56.0)$ & 0.024 \\
\hline Myalgia (\%) & $6(42.86)$ & $54(83.08)$ & $2(4.0)$ & $1(2.0)$ & $<0.001$ \\
\hline Malaise (\%) & $7(30.43)$ & $21(42.0)$ & $34(68.0)$ & $37(74)$ & 0.001 \\
\hline Nausea (\%) & $1(4.35)$ & $20(30.77)$ & $7(14.0)$ & $5(10.0)$ & 0.102 \\
\hline Diarrhea (\%) & $1(4.35)$ & $5(10.0)$ & $8(16.0)$ & $6(10.0)$ & 0.834 \\
\hline Fever (\%) & $1(4.35)$ & $2(4.0)$ & $4(8.0)$ & $1(2.0)$ & 0.240 \\
\hline
\end{tabular}




\begin{tabular}{llllll} 
Rash (\%) & 0 & $1(2.0)$ & $1(2.0)$ & 0 & 0.688 \\
\hline Somnolence (\%) & 0 & $1(2.0)$ & $2(4.0)$ & 0 & 0.421 \\
\hline Flu like symptoms (\%) & 0 & $1(2.0)$ & $2(4.0)$ & $2(4.0)$ & 0.738 \\
\hline Arthralgia (\%) & 0 & 0 & 0 & $1(2.0)$ & 0.480 \\
\hline Dizziness (\%) & $2(8.70)$ & $1(2.0)$ & 0 & 0 & 0.118 \\
\hline Paresthesia (\%) & 0 & 0 & 0 & 0 & - \\
\hline Vomiting (\%) & 0 & $3(6.0)$ & 0 & 0 & 0.057
\end{tabular}

Supplementary Table 2 The geometric mean anti-RBD IgG concentration (GMC) and the geometric mean ratio (GMR) between post boosting and baseline or post primary series*. 


\begin{tabular}{|c|c|c|c|c|c|}
\hline \multirow{2}{*}{$\begin{array}{l}\text { Anti-RBD IgG antibody } \\
\text { concentration (GMC) } \\
\text { CoronaVac-prime }(n=179)\end{array}$} & \multicolumn{5}{|c|}{ Type of booster vaccinations } \\
\hline & & & & & \\
\hline & BBIBP- & \multirow{3}{*}{$\begin{array}{l}\text { ChAdOx } 1 \\
n=65\end{array}$} & \multirow{3}{*}{$\begin{array}{l}30 \mu \mathrm{g} \\
\text { BNT162b2 } \\
\mathrm{n}=50\end{array}$} & $15 \mu \mathrm{g}$ & \multirow{3}{*}{$\begin{array}{l}p \text {-value } \\
\text { between } \\
\text { groups }\end{array}$} \\
\hline & & & & BNT162b2 & \\
\hline & $n=14$ & & & $\mathrm{n}=50$ & \\
\hline \multirow[t]{2}{*}{ GMC at baseline $(95 \% \mathrm{Cl})$} & 34.32 & 38.18 & 33.31 & 37.67 & \multirow[t]{2}{*}{0.7616} \\
\hline & $\begin{array}{l}(22.33 \\
52.77)\end{array}$ & $\begin{array}{l}(31.21 \\
46.71)\end{array}$ & $\begin{array}{l}(26.72 \\
41.53)\end{array}$ & $\begin{array}{l}(31.65, \\
44.84)\end{array}$ & \\
\hline \multirow[t]{2}{*}{ GMC after boosting $(95 \% \mathrm{Cl})$} & 154.6 & \multirow{2}{*}{$\begin{array}{l}1358.0 \\
(1141.84 \\
1615.07)\end{array}$} & \multirow{2}{*}{$\begin{array}{l}5152.2 \\
(4491.65 \\
5909.83)\end{array}$} & \multirow{2}{*}{$\begin{array}{l}3981.1 \\
(3397.15 \\
4665.42)\end{array}$} & \multirow[t]{2}{*}{$<0.0001$} \\
\hline & $\begin{array}{l}(92.11 \\
259.47)\end{array}$ & & & & \\
\hline \multirow{2}{*}{$\begin{array}{l}\text { GMR between post-boosting } \\
\text { and baseline }(95 \% \mathrm{Cl})\end{array}$} & \multirow{2}{*}{$\begin{array}{l}4.5(2.98, \\
6.80)\end{array}$} & \multirow{2}{*}{$\begin{array}{l}35.6(29.18 \\
43.34)\end{array}$} & 154.7 & & \multirow[t]{2}{*}{$<0.0001$} \\
\hline & & & $\begin{array}{l}(124.30 \\
192.50)\end{array}$ & $\begin{array}{l}\text { (90.31) } \\
123.68)\end{array}$ & \\
\hline $\begin{array}{l}\text { GMR between post-boosting } \\
\text { and post primary series of } \\
\text { CoronaVac*}\end{array}$ & $\begin{array}{l}0.94 \\
(0.53 \\
1.67)\end{array}$ & $\begin{array}{l}8.26(6.29 \\
10.85)\end{array}$ & $\begin{array}{l}31.34 \\
(24.37 \\
40.30)\end{array}$ & $\begin{array}{l}24.22 \\
(18.60 \\
31.54)\end{array}$ & $<0.0001$ \\
\hline \multicolumn{6}{|l|}{ ChAdOx1-prime $(n=173)$} \\
\hline & $\begin{array}{l}\text { BBIBP- } \\
\text { CorV }\end{array}$ & \multirow{3}{*}{$\begin{array}{l}\text { ChAdOx1 } \\
n=50\end{array}$} & \multirow{3}{*}{$\begin{array}{l}30 \mu \mathrm{g} \\
\text { BNT162b2 } \\
\mathrm{n}=49\end{array}$} & $15 \mu \mathrm{g}$ & \multirow{3}{*}{$\begin{array}{l}p \text {-value } \\
\text { between } \\
\text { groups }\end{array}$} \\
\hline & & & & BNT162b2 & \\
\hline & $n=23$ & & & $n=50$ & \\
\hline \multirow{2}{*}{$\begin{array}{l}\text { Geometric mean level at } \\
\text { baseline } \\
(95 \% \mathrm{Cl})\end{array}$} & 106.6 & 105.7 & 95.98 & 90.11 & \multirow[t]{2}{*}{0.7661} \\
\hline & $\begin{array}{l}(70.89 \\
160.29)\end{array}$ & $\begin{array}{l}(80.97 \\
137.97)\end{array}$ & $\begin{array}{l}(75.84 \\
121.45)\end{array}$ & $\begin{array}{l}(73.62 \\
110.30)\end{array}$ & \\
\hline $\begin{array}{l}\text { Geometric mean level after } \\
\text { boosting }(95 \% \mathrm{Cl})\end{array}$ & $\begin{array}{l}128.1 \\
(93.52 \\
175.37)\end{array}$ & $\begin{array}{l}246.4 \\
(199.59 \\
304.20)\end{array}$ & $\begin{array}{l}2363.8 \\
(2005.58 \\
2786.06)\end{array}$ & $\begin{array}{l}1961.9 \\
(1624.61 \\
2369.10)\end{array}$ & $<0.0001$ \\
\hline $\begin{array}{l}\text { GMR between post-boosting } \\
\text { and baseline }(95 \% \mathrm{Cl})\end{array}$ & $\begin{array}{l}1.2(1.01 \\
1.43)\end{array}$ & $\begin{array}{l}2.3(1.92 \\
2.83)\end{array}$ & $\begin{array}{l}25.1(20.30 \\
31.01)\end{array}$ & $\begin{array}{l}21.8(18.28 \\
25.92)\end{array}$ & $<0.0001$ \\
\hline $\begin{array}{l}\text { GMR between post-boosting } \\
\text { and post primary series of } \\
\text { ChAdOX } 1^{\star}\end{array}$ & $\begin{array}{l}0.46 \\
(0.28 \\
0.65)\end{array}$ & $\begin{array}{l}0.88(0.58 \\
1.13)\end{array}$ & $\begin{array}{l}8.49(5.71 \\
10.44)\end{array}$ & $\begin{array}{l}7.04(4.69 \\
8.84)\end{array}$ & $<0.0001$ \\
\hline
\end{tabular}

*The post primary series GMC was derived from the study in the same setting as the current study (Reference: Angkasekwinai, N. et al. Safety and Immunogenicity of CoronaVac and ChAdOx1 Against the SARS-CoV-2 Circulating Variants of Concern (Alpha, Delta, Beta) in Thai Healthcare Workers. Preprint at 
medRxiv https://doi:10.1101/2021.10.03.21264451 (2021)). The post primary series GMC (95\% Cl) at 2 weeks after the second dose of the 2-dose homologous CoronaVac, 4 weeks apart, was 164.4 (133.55, 202.43); and after 2-dose homologous ChAdOx1,10 weeks apart, was 278.5 (195.66,396.33).

\section{Figures}

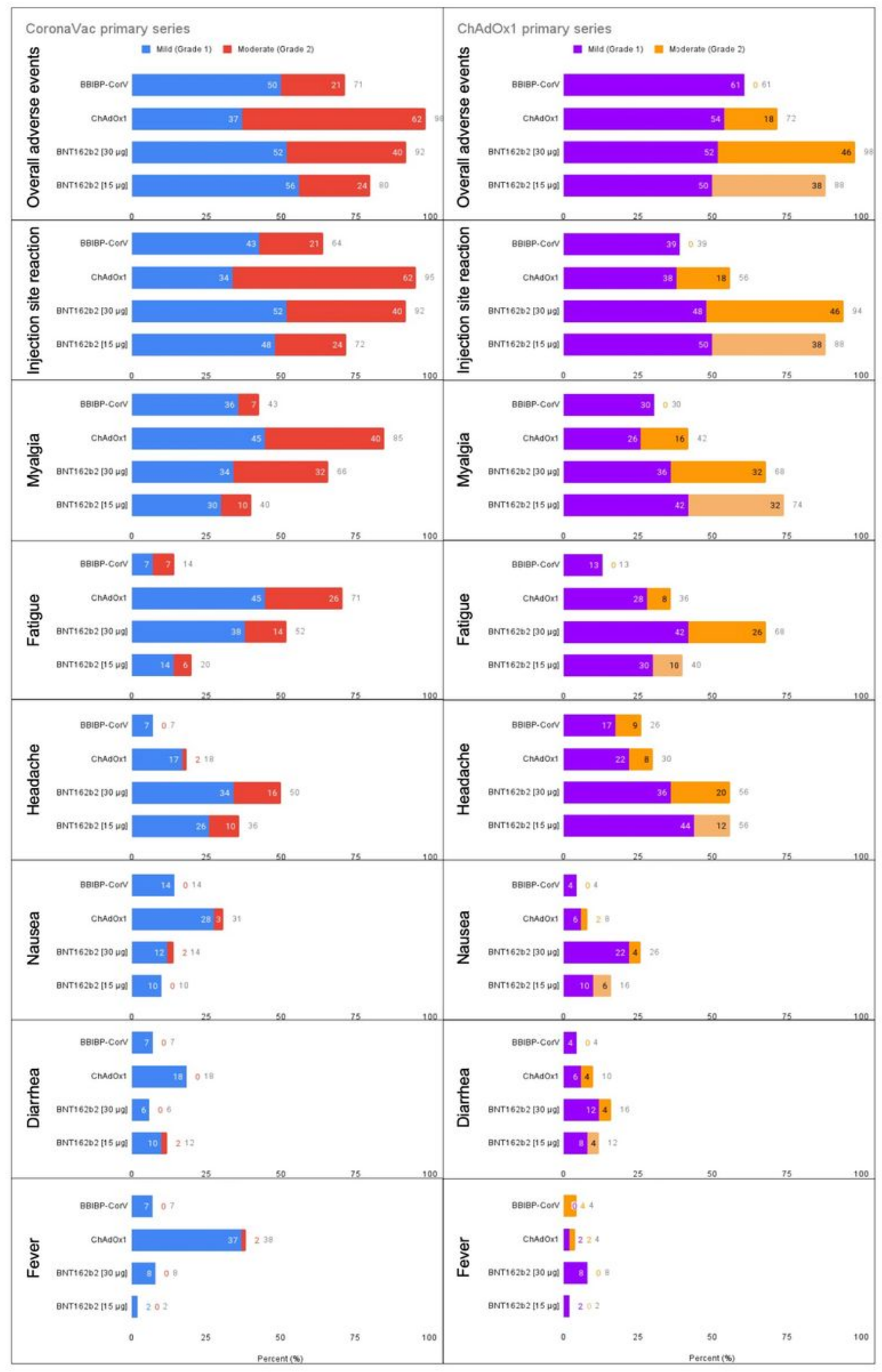

Figure 1 
Adverse events following four different booster vaccinations. The stacked bars showed the percentage of participants who reported mild and moderate adverse events after the booster vaccinations in the subjects who had received 2-dose CoronaVac-primary series (A) and ChAdOx1-primary series (B) vaccination. Chi-square was used for statistical analyses and the $p$ values were shown on the graphs.

\section{Figure 2}

Quantitation of anti-SARS-CoV-2 RBD IgG after booster vaccination. The scatter dot plot demonstrates the anti-SARS-CoV-2 RBD IgG concentration before and 2 weeks after different booster vaccination in the plasma of study subjects who had received 2-doses CoronaVac primary series (A) or ChAdOx1-primary series (B) 8-12 weeks prior. The numbers in the graph represent geometric mean and the error bars represent $95 \%$ confidence interval. The number of tested samples were indicated for each type for booster vaccine. The upper dotted line represents the geometric mean concentration (GMC) of anti-SARS-CoV-2 RBD IgG at 2 weeks after the second dose of the 2-dose primary series 4 weeks apart of CornaVac (A) and 8 weeks apart of ChAdOx1 (B) from reference Angkasekwinai N, et al. medRxiv preprint doi: https://doi.org/10.1101/2021.10.03.21264451, and the lower dot line represents the cut-off level for seropositivity. Indicated $\mathrm{p}$ values were for the unpair $\mathrm{t}$ test comparison between different types of booster vaccine and the pair $t$ test comparison between before and after boost. The value of $11,360 \mathrm{BAU} / \mathrm{mL}$ was used for any samples that were reported $>11,360 \mathrm{BAU} / \mathrm{mL}$ by the machine. The scatter dot plot demonstrates the level of anti-SARS-CoV-2 RBD IgG at 2 weeks after various booster vaccination in the plasma of study subjects who had completed 2-doses of CoronaVac primary series (blue) or ChAdOx1 primary series (red) (C). The numbers in the graph represent GMC and the error bars represent $95 \%$ confidence interval. The number of tested samples were indicated for each type for booster vaccine. Unpair $t$ test was used to compare between each group with indicated $p$ value.

\section{Figure 3}

Plaque reduction neutralisation test (PRNT50) titres for SARS-CoV-2 delta and beta variants after different booster vaccines. Scatter dot plots demonstrate PRNT50 titre against the (A) delta or (B) beta strain at 2 weeks after different booster vaccines in the study subjects who had completed two doses of Coronavac or ChAdOx1 for 8-12 weeks. The PRNT50 titre of 1:5 was used for all that were below the detectable level $(<1: 10)$. The numbers in the graph represent geometric mean titre (GMT) and the error bars represent $95 \%$ confidence interval $(\mathrm{Cl})$. The upper dotted line represents the geometric mean values of anti-SARS-CoV-2 RBD $\operatorname{lgG}$ at 2 weeks after the second dose of the 2-dose primary series 4 weeks apart of CornaVac (A) and 8 weeks apart of ChAdOx1 (B) from reference Angkasekwinai N, et al. medRxiv preprint doi: https://doi.org/10.1101/2021.10.03.21264451; and the lower dot line represents the cut-off level for seropositivity. Unpaired t tests were used for statistical analyses and the $p$ values were indicated. (C) comparison of the plaque reduction neutralisation test (PRNT50) titres for the SARS-CoV-2 delta and beta variants after different booster vaccine in among each primary series. Aligned dot plots show the 
PRNT50 titre against the delta (teal) or beta (pink) variant in the plasma of study subjects who had completed two doses of CoronaVac or ChAdOx1 at 2 weeks after booster with BBIBP-CorV, ChAdOX1, 30 $\boldsymbol{\mu g}$. BNT162b2 and $15 \boldsymbol{\mu g}$. BNT162b2. The PRNT50 titre of 1:5 was used for all that were below the detectable level $(<1: 10)$. Ratio paired $t$ test was used for statistical analyses.

\section{Figure 4}

Correlation between the level of anti-SARS-CoV-2 RBD IgG and plaque reduction neutralisation test (PRNT50) titres for the SARS-CoV-2 delta and beta variants. Dot plots show the correlation between the level of anti-SARS-CoV-2 RBD IgG and PRNT50 titre against the $(A, B)$ delta or $(C, D)$ beta variant in the plasma of study subjects who had completed two doses of CoronaVac or ChAdOx 1 at 2 weeks after booster with BBIBP-CorV (green), ChAdOX1 (red), $30 \mu \mathrm{g}$. BNT162b2 (orange) and $15 \boldsymbol{\mu g}$. BNT162b2 (yellow). The PRNT50 titre of 1:5 was used for all that were below the detectable level ( $<1: 10)$. Pearson's correlation coefficient $(r)$ with $p$ value for each booster vaccine was indicated.

\section{Supplementary Files}

This is a list of supplementary files associated with this preprint. Click to download.

- FigureS1BoosterCOVIDforNature29Nov21.png.png 